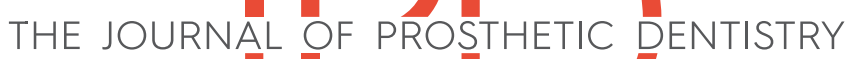

CLINICAL RESEARCH

\section{Color stability of ceramic laminate veneers cemented with light-polymerizing and dual-polymerizing luting agent: A split-mouth randomized clinical trial}

\author{
Ana Maria Estivalete Marchionatti, DDS, MSD, ${ }^{a}$ Vinícius Felipe Wandscher, DDS, MSD, ${ }^{b}$ \\ Michele Mirian May, DDS, MSD, ${ }^{c}$ Marco Antonio Bottino, DDS, MSD, PhD, and \\ Liliana Gressler May, DDS, MSD, PhD
}

Dental ceramics are widely used for esthetic restorative treatments owing to their desirable characteristics: color stability, translucency, tooth-like optical properties, mechanical resistance, durability, and compatibility with periodontal tissue. ${ }^{1-4}$ A popular approach for esthetic treatments is the use of laminate veneers, which are indicated to increase tooth size, reduce diastemas, and to correct form and discoloration. ${ }^{1,5}$ With the evolution of adhesive systems, resin cements and ceramic materials, it is now possible to attach 0.1- to 0.7-mm-thick ceramic laminate veneers with minimal or no tooth preparation. ${ }^{1,3,6-8}$

The color stability of the luting agents influences the esthetic result of ceramic restorations, which is a determinant of long-term success $^{3,6,9}$ especially in highly translucent restorations. ${ }^{10,11}$ Any alteration of the cement color that results in a

\begin{abstract}
Statement of problem. The color stability of luting agents influences the esthetics of ceramic laminate veneers. Clinical studies that have evaluated the color changes of veneers cemented to enamel with light- and dual-polymerizing resin cement are lacking.

Purpose. The purpose of this split-mouth randomized clinical trial was to evaluate the color change and marginal discoloration of dual- and light-polymerizing cement used for cementation of ceramic laminate veneers.

Material and methods. In 10 participants, $0.3-\mathrm{mm}$-thick ceramic laminate veneers were cemented on the buccal surface of the second premolars without tooth preparation. A randomized application of light-polymerized cement was used on one side and a dual-polymerized cement on the contralateral side. The operator and participants were blinded to the activation mode. Color was evaluated by a blinded evaluator with a spectrophotometer at 24 hours and at 2, 6, 12, and 24 months after cementation. The CIELab $\left(\Delta \mathrm{E}^{*}{ }_{\mathrm{ab}}\right)$ and $\mathrm{CIEDE} 2000\left(\Delta \mathrm{E}^{*}{ }_{00}\right)$ formulas were used to quantify color alteration, and $\Delta \mathrm{a}^{*}, \Delta \mathrm{b}^{*}$, and $\Delta \mathrm{L}^{*}$ were calculated between the first and subsequent measurements. US Public Health Service guidelines were used to evaluate the marginal discoloration.
\end{abstract}

Results. Wilcoxon tests did not show a statistical difference in $\Delta \mathrm{E}^{*}{ }_{\mathrm{ab}}$ and $\Delta \mathrm{E}^{*}{ }_{00}$ between the groups $(P>.05)$. At 24 months, the median $\Delta \mathrm{E}^{*}$ ab was 2.31 (interquartile ranges [IQR]: 3.34) for the lightpolymerizing mode and 1.57 (IQR: 0.41 ) for the dual-polymerizing mode, while the median $\Delta \mathrm{E}_{00}^{*}$ was 1.65 for the light-polymerizing mode (IQR: 2.34) and 1.18 for the dual-polymerizing mode (IQR: 0.25). The thresholds for clinically acceptable color changes $\Delta \mathrm{E}^{*}{ }_{\mathrm{ab}}>3.46$ and $\Delta \mathrm{E}^{*}{ }_{00}>2.25$ were found for both curing modes. Marginal discoloration was observed from the 2-year assessment.

Conclusions. The color stability of ceramic laminate veneers was similar for both of the polymerizing modes for all evaluated periods. Marginal discoloration increased over a 2-year period for both the light- and the dual-polymerizing modes. (J Prosthet Dent 2017;118:604-610)

mismatch with the adjacent teeth is a reason for restoration replacement ${ }^{6,12}$ and may be considered a treatment failure. ${ }^{12}$

\footnotetext{
${ }^{a}$ Doctoral student, Department of Restorative Dentistry, Faculty of Dentistry, Federal University of Santa Maria, Rio Grande do Sul, Brazil.

${ }^{b}$ Doctoral student, Department of Restorative Dentistry, Faculty of Dentistry, Federal University of Santa Maria, Rio Grande do Sul, Brazil.

'Staff dentist, Department of Restorative Dentistry, Faculty of Dentistry, Federal University of Santa Maria, Rio Grande do Sul, Brazil.

${ }^{\mathrm{d}}$ Titular Professor, Department of Restorative Dentistry, São José dos Campos Dental School, São Paulo State University, São Paulo, Brazil.

${ }^{\text {e} A d j u n c t ~ P r o f e s s o r, ~ D e p a r t m e n t ~ o f ~ R e s t o r a t i v e ~ D e n t i s t r y, ~ F a c u l t y ~ o f ~ D e n t i s t r y, ~ F e d e r a l ~ U n i v e r s i t y ~ o f ~ S a n t a ~ M a r i a, ~ R i o ~ G r a n d e ~ d o ~ S u l, ~ B r a z i l . ~}$
} 


\section{Clinical Implications}

Clinicians should consider that color changes beyond the threshold of clinical acceptability and slight marginal discoloration are observed for a portion of the veneers regardless of the activation mode of the cement (light- or dual-polymerized).

The luting agents available for ceramic restorations can be auto-, light-, or dual-polymerizing resin cement. In vitro studies have indicated that dual-polymerizing resin cements undergo greater color alteration than light-polymerizing cements, which is usually attributed to the oxidation of aromatic tertiary amines present on the dual cements as accelerators of the autopolymerizing reaction. ${ }^{1,9,12-17}$ The presence of unreacted doublebonds, composition of monomers used in the matrix, filler size and content, water absorption, and environmental factors may also cause color instability in resin cements. ${ }^{2,18-20}$

Artificially accelerated aging and water storage may help predict cement color changes over time. However, in clinical situations, the restorative materials are subjected to numerous dynamic conditions in the oral environment, including temperature variations, continuous humidity, food colorants, and mechanical loading.7,18 The authors are unaware of published clinical studies that have assessed the color change of the resin cements used for the adhesion of ceramic laminate veneers.

The purpose of this randomized clinical trial was to evaluate the color alteration and marginal discoloration of a dual-polymerizing and a light-polymerizing cement used to cement ultrathin ceramic laminate veneers. The hypotheses were that the polymerizing mode would show no influence on color stability and that the use of light- or dual-polymerizing cement would not influence marginal discoloration.

\section{MATERIAL AND METHODS}

This double-blind, split-mouth randomized clinical trial was approved by the Ethical Committee (CAAE: 13408513.9.0000.5346) and performed in accordance with the Consolidated Standards of Reporting Trials (CONSORT) statement (protocol is available at http:// www.ensaiosclinicos.gov.br/rg/RBR-25rc6q/). Written informed consent was obtained from the 10 participants included in the study. All participants were postgraduate Oral Science students who met the following inclusion criteria: good general and oral health; absence of orthodontic appliances; absence of posterior reverse articulation; absence of noncarious cervical lesions; vital maxillary second premolars without restorations and with color homogeneity in relation to adjacent teeth; and a minimum 6-mm diameter for the area of the buccal surface of the maxillary second premolars (slightly larger than the $5 \mathrm{~mm}$ spectrophotometer tip). Participants with the following characteristics were excluded from the study: gingival recession on the buccal surface of the maxillary second premolars; dental sensitivity to air blast; enamel or dentin defects; tetracycline staining; unilateral or bilateral reverse articulation; mandibular prognathism; maxillary retrognathism; and smokers. Sample size calculation was performed based on a color difference of 3.0 between the means of the groups and a standard deviation of $\pm 1.7 .{ }^{15}$ Significance level and statistical power were defined as $5 \%$ and $80 \%$, respectively, and a possible loss to follow-up was taken into consideration.

Following clinical and radiographic examination and cold pulp sensitivity testing to verify vitality, the participants answered a questionnaire adapted from Hedric et $\mathrm{al}^{21}$ regarding the intake frequency of potentially pigmenting food and beverages. The questionnaire was validated by comparison with 7-day dietary intake records, using weighted kappa to verify whether participants' answers reflected their habits. The results and validity assessment of the questionnaire are displayed in Table 1. Agreement between the questionnaires and dietary records was perfect $(K=1)$ or almost perfect $(K>0.81)$ for all items, except for tomato sauce, which was substantial $(\mathrm{K}=0.80)$, and for colored juices, which was moderate $(\mathrm{K}=0.47) .{ }^{22}$

No tooth preparation was performed. Maxillary arch impressions were made with polyvinyl siloxane (Express; 3M ESPE) with the double-mix technique, and Type IV gypsum (GC Fujirock EP; GC America) casts were obtained. Two 0.3-mm-thick laminate veneers were fabricated by a single dental technician for the buccal surface of the participants' left and right maxillary second premolars, using a high-translucency lithium disilicate glass-ceramic (IPS e.max Press, shade B1; Ivoclar Vivadent $A G)$. The laminate veneers were placed $0.5 \mathrm{~mm}$ from the gingival margin, following the anatomy of the teeth and extending slightly to the proximal and occlusal surfaces without interfering with the proximal contacts or occlusion. The margins were beveled to have finish lines at the tooth level without overcontouring. The second premolars were selected because they are in a posterior area but are still visible and accessible for color evaluation.

The polymerization mode was randomized for each side according to a software-generated randomization list (Random.org Integer Generator; http://www.random. org). Randomization was concealed by sequentially numbered, opaque, sealed envelopes containing the polymerization mode (light- or dual-polymerizing) and side (left or right). The envelopes were prepared by an investigator (V.F.W.) with no involvement in the trial. For each patient, a researcher (M.M.M.) who was not involved in the cementation and evaluation opened the envelope 
Table 1. Intake frequency of potentially pigmenting food and beverages and validity assessment ${ }^{\mathrm{a}}$

\begin{tabular}{|c|c|c|c|c|c|c|c|c|c|}
\hline Food or Beverage Type & Never & $\begin{array}{l}\text { Less than Once } \\
\text { per Week }\end{array}$ & $\begin{array}{l}\text { Once per } \\
\text { Week }\end{array}$ & $\begin{array}{l}\text { 2-3 Times } \\
\text { per Week }\end{array}$ & $\begin{array}{l}\text { 4-6 Times } \\
\text { per Week }\end{array}$ & $\begin{array}{l}\text { Once per } \\
\text { Day }\end{array}$ & $\begin{array}{l}\text { Twice per } \\
\text { Day }\end{array}$ & $\begin{array}{l}\text { 3+ Times } \\
\text { per Day }\end{array}$ & $\begin{array}{l}\text { Kappa } \\
\text { Value }\end{array}$ \\
\hline Coffee & 2 & 0 & 0 & 2 & 1 & 2 & 2 & 1 & 0.84 \\
\hline Dark beer & 8 & 2 & 0 & 0 & 0 & 0 & 0 & 0 & 1 \\
\hline Black tea & 8 & 0 & 1 & 0 & 0 & 0 & 1 & 0 & 0.91 \\
\hline Colored tea & 3 & 2 & 1 & 3 & 1 & 0 & 0 & 0 & 0.87 \\
\hline Mate $^{b}$ & 1 & 1 & 1 & 1 & 2 & 1 & 3 & 0 & 0.92 \\
\hline Cola-based soda & 1 & 0 & 1 & 6 & 2 & 0 & 0 & 0 & 0.80 \\
\hline Colored soda & 4 & 4 & 0 & 2 & 0 & 0 & 0 & 0 & 0.89 \\
\hline Colored juice & 2 & 3 & 0 & 4 & 0 & 1 & 0 & 0 & 0.47 \\
\hline Coffee cough drop & 9 & 0 & 0 & 1 & 0 & 0 & 0 & 0 & 0.85 \\
\hline Colored cough drop & 7 & 2 & 0 & 1 & 0 & 0 & 0 & 0 & 0.90 \\
\hline Beet & 5 & 0 & 1 & 4 & 0 & 0 & 0 & 0 & 0.92 \\
\hline Chocolate & 1 & 1 & 0 & 6 & 1 & 1 & 0 & 0 & 0.84 \\
\hline Raw carrot & 2 & 2 & 2 & 3 & 0 & 1 & 0 & 0 & 0.88 \\
\hline Gelatin & 3 & 6 & 1 & 0 & 0 & 0 & 0 & 0 & 1 \\
\hline Molasses & 9 & 1 & 0 & 0 & 0 & 0 & 0 & 0 & 1 \\
\hline Colored medicines & 9 & 1 & 0 & 0 & 0 & 0 & 0 & 0 & 1 \\
\hline Saffron sauce & 9 & 1 & 0 & 0 & 0 & 0 & 0 & 0 & 0.91 \\
\hline Tomato sauce & 0 & 3 & 1 & 5 & 0 & 1 & 0 & 0 & 0.80 \\
\hline Colored grape & 7 & 3 & 0 & 0 & 0 & 0 & 0 & 0 & 1 \\
\hline Red wine & 6 & 4 & 0 & 0 & 0 & 0 & 0 & 0 & 1 \\
\hline
\end{tabular}

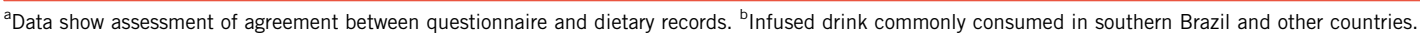

and informed the operator (L.G.M.) of the cementation mode and side. The intaglios of the laminate veneers were treated with 10\% hydrofluoric acid (Condicionador de Porcelanas; Dentsply Sirona) and a silane (Monobond S; Ivoclar Vivadent AG). Before cementation, the maxillary second premolars were isolated, the enamel pumiced (Pedra Pomes; Biodinâmica), and the gingival displacement cord (Retraflex retraction cord 000; Biodinâmica) placed in the gingival sulcus. The tooth was etched with $37 \%$ phosphoric acid (Condac 37; FGM), rinsed, and dried. The adjacent teeth were isolated with polytetrafluoroethylene tape (Polyfita; Seal Tape), and the lightpolymerizing adhesive (Tetric N Bond; Ivoclar Vivadent AG) was applied and photoactivated (Radii-cal; SDI) for 20 seconds. The laminate veneers were cemented with the transparent shade of cement (Variolink II; Ivoclar Vivadent AG) (Tables 2, 3). One side underwent dualpolymerization (using both the base and the catalyst in a 1:1 ratio), whereas the contralateral side underwent light-polymerization (using only the base) (Fig. 1A). Photoactivation was performed for 60 seconds on both groups. The same researcher (M.M.M.) that opened the envelope spatulated the cement and handed it to the operator (L.G.M.) so that the participant and operator were blinded to the polymerization mode. The second cementation was performed on the contralateral side with the remaining polymerization mode.

Color and marginal staining were evaluated at 24 hours (baseline) and at 2, 6, 12, and 24 months after cementation by a blinded evaluator (A.M.E.M.), using a clinical spectrophotometer (Vita Easyshade; Vita Zahnfabrik).
Table 2. Variolink II chemical composition

\begin{tabular}{ll}
\hline Base Plus Catalyst & \multicolumn{1}{c}{ Composition } \\
\hline Monomer & $\begin{array}{l}\text { Bis-phenol-A-diglycidylmethacrylate (Bis-GMA); } \\
\text { Urethane dimethacrylate (UDMA); triethyleneglycol } \\
\text { dimethacrylate (TEGDMA) }\end{array}$ \\
\hline Inorganic fillers & $\begin{array}{l}\text { Barium glass, ytterbium trifluoride, barium and } \\
\text { aluminium fluorosilicate glass and spheroid mixed oxide }\end{array}$ \\
\hline Additional contents & Benzoyl peroxide, catalysts, stabilizers and pigments. \\
\hline
\end{tabular}

Table 3. Variolink II composition by weight (\%)

\begin{tabular}{lcc}
\hline Composition & Base & Catalyst \\
\hline Dimethacrylates & 26.3 & 22.0 \\
\hline Inorganic fillers & 73.4 & 77.2 \\
\hline Catalysts and stabilizers & 0.3 & 0.8 \\
\hline Pigments & $<0.1$ & $<0.1$ \\
\hline
\end{tabular}

Individualized light-polymerizing acrylic resin guides (Elite LC Tray; Zhermack) with a 6-mm-diameter window were positioned on the buccal surface of the second premolars to standardize the positions for the color evaluations (Fig. 1B). The color measurements were recorded in the CIELab system, which defines color on 3 axes: $L^{*}$, lightness, ranging from 0 (black) to 100 (white); and $a^{*}$ and $\mathrm{b}^{*}$, chromatic characteristics ranging from red $\left(+\mathrm{a}^{*}\right)$ to green $\left(-\mathrm{a}^{*}\right)$ and yellow $\left(+\mathrm{b}^{*}\right)$ to blue $\left.\left(-\mathrm{b}^{*}\right)\right)^{7,16,23-25}$ The color was measured 3 times, and the mean $L^{*}, a^{*}$, and $b^{*}$ values were calculated. The variation of each coordinate between the first and subsequent measurements was calculated as follows: $\left[\Delta \mathrm{L}^{*}=\mathrm{L}^{*}\right.$ final $-\mathrm{L}^{*}$ initial $]$, $\left[\Delta \mathrm{a}^{*}=\mathrm{a}^{*}\right.$ final $-\mathrm{a}^{*}$ initial $]$, and $\left[\Delta \mathrm{b}^{*}=\mathrm{b}^{*}\right.$ final $-\mathrm{b}^{*}$ initial $]$. 

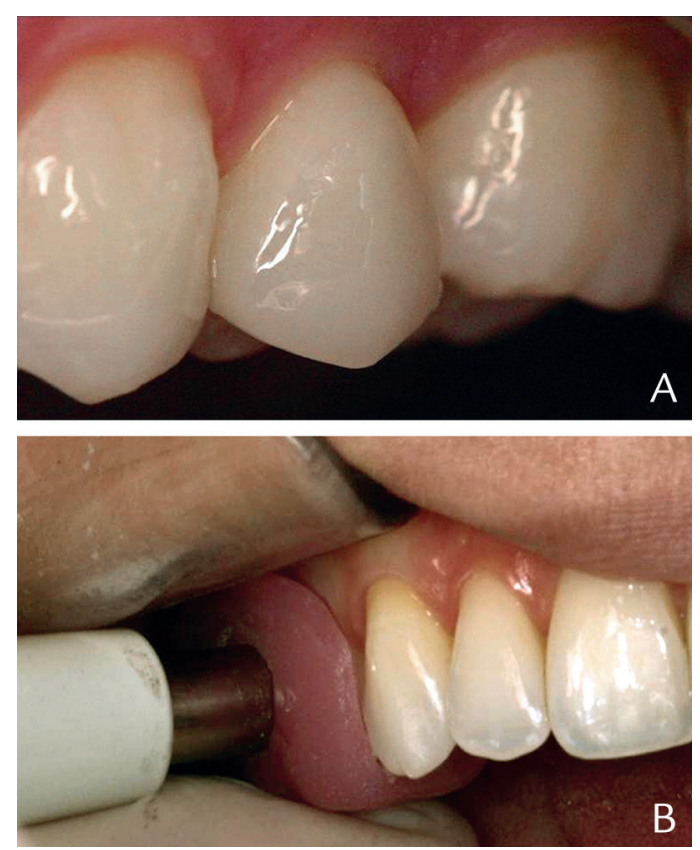

Figure 1. A, Ceramic laminate veneer after cementation. B, Guide for color measurement: window placed on buccal surface to ensure reproducibility of spectrophotometer readings.

The CIELab color change $\left(\Delta \mathrm{E}^{*} \mathrm{ab}\right)$ was calculated according to the following formula ${ }^{1}$ :

$\Delta \mathrm{E}_{\mathrm{ab}}^{*}=\left[\left(\Delta \mathrm{L}^{*}\right)^{2}+\left(\Delta \mathrm{a}^{*}\right)^{2}+\left(\Delta \mathrm{b}^{*}\right)^{2}\right]^{1 / 2}$

Color differences were also calculated using the CIEDE2000 formula as follows ${ }^{26}$ :

$$
\begin{gathered}
\Delta \mathrm{E}_{00}^{*}=\left[\left(\Delta \mathrm{L}^{\prime} / \mathrm{k}_{\mathrm{L}} \mathrm{S}_{\mathrm{L}}\right)^{2}+\left(\Delta \mathrm{C}^{\prime} / \mathrm{k}_{\mathrm{C}} \mathrm{S}_{\mathrm{C}}\right)^{2}+\left(\Delta \mathrm{H}^{\prime} / \mathrm{k}_{\mathrm{H}} \mathrm{S}_{\mathrm{H}}\right)^{2}\right. \\
\left.+\mathrm{R}_{\mathrm{T}}\left(\Delta \mathrm{C}^{\prime} / \mathrm{k}_{\mathrm{C}} \mathrm{S}_{\mathrm{C}}\right)\left(\Delta \mathrm{H}^{\prime} / \mathrm{k}_{\mathrm{H}} \mathrm{S}_{\mathrm{H}}\right)\right]^{1 / 2}
\end{gathered}
$$

where $\Delta \mathrm{L}^{\prime}, \Delta \mathrm{C}^{\prime}$, and $\Delta \mathrm{H}^{\prime}$ are the differences in lightness, chroma, and hue, respectively, between the baseline and the subsequent color readings; $\mathrm{R}_{\mathrm{T}}$ is the rotation function corresponding to chroma and hue difference interaction in the blue region; $\mathrm{S}_{\mathrm{L}}, \mathrm{S}_{\mathrm{C}}$ and $\mathrm{S}_{\mathrm{H}}$ are weighting terms for adjustment of the total color difference for variation in perceived magnitude with variation in the location of the color coordinate difference between 2 color measurements; and $\mathrm{k}_{\mathrm{L}}, \mathrm{k}_{\mathrm{C}}$, and $\mathrm{k}_{\mathrm{H}}$ are correction terms for the experimental conditions. ${ }^{13}$

Marginal staining was classified according to modified U.S. Public Health Service guidelines ${ }^{27}$ : Alfa, absence of marginal discoloration; Bravo, discoloration on less than half of the circumferential margin; and Charlie, discoloration on more than half of the circumferential margin. ${ }^{28}$

The normal distribution and homoscedasticity of the data were investigated using the Shapiro-Wilk and
Table 4. Mean \pm SD CIELab values for ceramic laminate veneers luted with light-polymerizing and dual-polymerizing cement

\begin{tabular}{lcccc}
\hline Cement & 2 months & $\mathbf{6}$ months & $\mathbf{1 2}$ months & 24 months \\
\hline$\Delta \mathrm{L}^{*}$ & & & & \\
\hline Light-polymerizing & $-0.13( \pm 3.43)$ & $-0.62( \pm 2.65)$ & $0.84( \pm 2.74)$ & $0.06( \pm 3.5)$ \\
\hline Dual-polymerizing & $-1.15( \pm 3.47)$ & $-1.43( \pm 1.37)$ & $0.45( \pm 3.48)$ & $0.02( \pm 2.6)$ \\
\hline$\Delta \mathrm{a}^{*}$ & & & & \\
\hline Light-polymerizing & $0.12( \pm 0.28)$ & $-0.01( \pm 0.23)$ & $0.30( \pm 0.35)$ & $0.01( \pm 0.36)$ \\
\hline Dual-polymerizing & $0.33( \pm 0.43)$ & $0.16( \pm 0.42)$ & $0.63( \pm 0.35)$ & $0.3( \pm 0.41)$ \\
\hline$\Delta \mathrm{b}^{*}$ & & & & \\
\hline Light-polymerizing & $-0.01( \pm 0.99)$ & $-0.52( \pm 1.62)$ & $0.32( \pm 1.24)$ & $0.48( \pm 1.6)$ \\
\hline Dual-polymerizing & $-0.05( \pm 1.72)$ & $-0.20( \pm 1.55)$ & $0.40( \pm 1.30)$ & $1.05( \pm 1.43)$ \\
\hline
\end{tabular}

Levene tests. After the normal distribution and equality of variances were verified, $\Delta \mathrm{L}^{*}, \Delta \mathrm{a}^{*}$, and $\Delta \mathrm{b}^{*}$ were subjected to paired $t$ tests. Because not all data had a normal or homoscedastic distribution, the medians and interquartile ranges (IQR) were calculated for $\Delta \mathrm{E}^{*}{ }_{\mathrm{ab}}$ and $\Delta \mathrm{E}^{*}{ }_{00}$, and a nonparametric Wilcoxon test was performed. Marginal discoloration was compared using a McNemar test ( $\alpha=.05$ for all tests).

\section{RESULTS}

No participant was lost to follow-up. No statistical differences were found between the light-polymerizing and dual-polymerizing groups for all the studied parameters across the evaluated periods $(P>.05)$. Table 4 shows $\Delta \mathrm{L}^{*}$, $\Delta \mathrm{a}^{*}$, and $\Delta \mathrm{b}^{*}$ values for each period. At 24 months, the median $\Delta \mathrm{E}^{*}$ ab was 2.31 (IQR: 3.34, maximum: 5.72, minimum: 0.34) for the light-polymerizing mode and 1.57 (IQR: 0.41, maximum nonoutlier: 1.80, minimum: 1.11) for the dual-polymerizing mode; while the median $\Delta \mathrm{E}^{*}{ }_{00}$ was 1.65 for the light-polymerizing mode (IQR: 2.34, maximum: 3.76, minimum: 0.37) and 1.18 (IQR: 0.25 , maximum non-outlier: 1.25 , minimum: 0.76 ) for the dual-polymerizing mode. Figure 2 shows the boxplots for both groups in each evaluated period.

$\Delta \mathrm{E}^{*}{ }_{\mathrm{ab}}$ values $\geq 3.46$ and $\Delta \mathrm{E}^{*}{ }_{00}$ values $\geq 2.25$ were considered clinically unacceptable. ${ }^{23}$ At 24 months, 4 teeth from the light-polymerizing group and 2 teeth from the dual-polymerizing group showed an unacceptable color change for both the $\Delta \mathrm{E}^{*}{ }_{\mathrm{ab}}$ and $\Delta \mathrm{E}^{*}{ }_{00}$ thresholds.

No marginal staining was found in either group until the 6-month follow-up (all restorations were classified as Alfa). At the 12-month evaluation, 2 restorations were classified as Bravo (1 in each group), and, at 24 months, 7 restorations (3 in the light- and 4 in the dualpolymerizing group) were classified as Bravo. The McNemar test did not show a statistical difference in marginal discoloration between the groups $(P>.05)$ at the 24 -month evaluation. Also, no statistical differences were found between baseline and 24-month marginal discoloration for each group $(P>.05)$. 

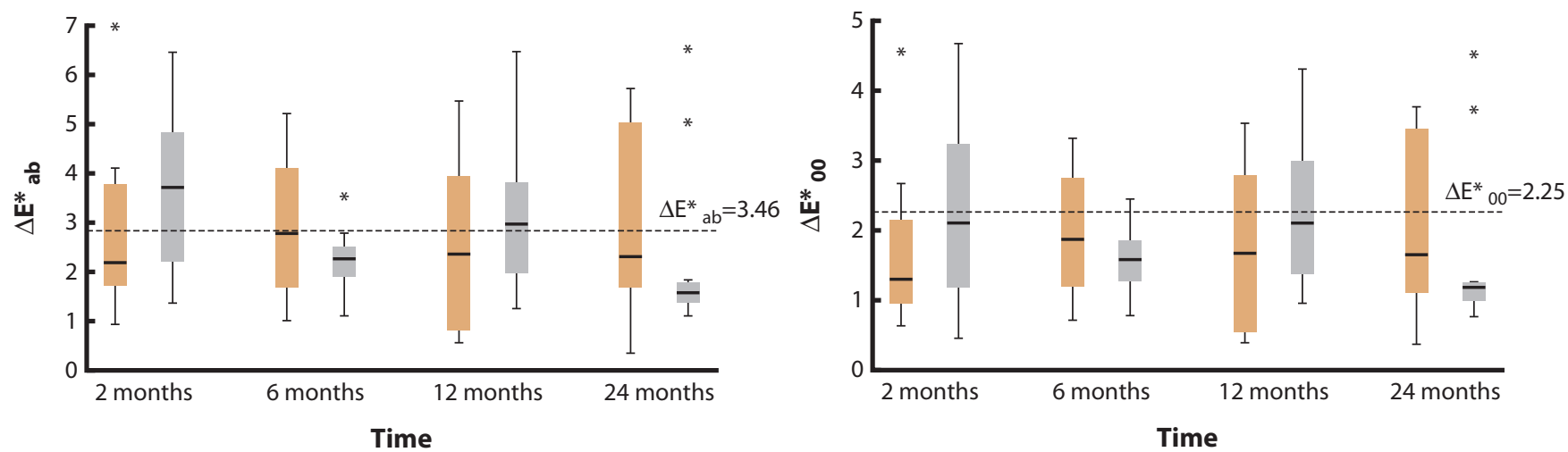

Light-polymerizing

Dual-polymerizing

Figure 2. $\Delta \mathrm{E}^{*}{ }_{\mathrm{ab}}$ and $\Delta \mathrm{E}^{*}{ }_{00}$ box plots show median, first and third quartiles, and minimum and maximum values. Outliers are marked as asterisks.

\section{DISCUSSION}

The present results support acceptance of the first hypothesis as the polymerization mode did not influence color alteration throughout the evaluated periods. Marginal discoloration was not significantly different between the light- and dual-polymerizing groups, so the second hypothesis was accepted.

The results are in accordance with those of other studies that used the same cement and also did not find a difference of color alterations between the dual- and light-polymerizing polymerization modes. ${ }^{6,7,12}$ However, these findings contradict those of $\mathrm{Lu}$ and Powers, ${ }^{16}$ who observed that the cement experienced a significant color alteration with dual- and light-polymerizing modes. However, cement thickness in that study was $2 \mathrm{~mm}$, much thicker than used clinically, and the cement was not covered by a ceramic layer, which could affect the color change. Furthermore, artificially accelerated aging was performed for $450 \mathrm{~kJ} / \mathrm{m}^{2}$, so the specimens were submitted to aggressive conditions of humidity, ultraviolet irradiation, and high temperature.

Dual-resin cements are thought to be more susceptible to color change because of the oxidation of aromatic tertiary amines present in their composition, whereas the higher color stability of light-polymerizing cements is because their component aliphatic amines are less susceptible to the oxidation process. ${ }^{1}$ For the Variolink II initiator-activator polymerization system, the base contains aliphatic and aromatic amines, while the catalyst contains benzoyl peroxide that reacts with the aromatic amines to produce chemical polymerization. ${ }^{6,7,16}$ According to Ghavam et $\mathrm{al}_{1}{ }^{6}$ when the base is used by itself for light polymerization only, the aromatic amines have no contact with the benzoyl peroxide present on the catalyst and thus remain intact, but the cement with each polymerization mode contains both amines, which may explain why no differences between the groups were found. 6,7
Although most color alteration result from oxidation of the amines, cement discoloration may also occur because of extrinsic factors such as environmental conditions, ultraviolet irradiation, humidity, heat, and food pigments and intrinsic factors such as matrix composition, filler size and content, and percentage of residual double bonds. ${ }^{18}$ The resin matrix of the cement used in the present study is composed of bisphenol A-glycidyl methacrylate (BisGMA), urethane dimethacrylate (UDMA), and triethylene glycol dimethacrylate (TEGDMA). Because UDMA is less susceptible to color change than other monomers because of lower rates of water sorption, some studies associate the stability of the cement color with the presence of UDMA and a reduction in the quantity of TEGDMA (a monomer with increased water uptake). ${ }^{17,18,29}$ In addition, when light transmission is limited, the resin cements undergo incomplete polymerization. The presence of incompletely converted monomer may explain cement discoloration. Therefore, color stability may also be associated with laminate veneers of high translucency and extreme thinness, which would facilitate complete cement polymerization. ${ }^{7,18}$ Another reason for the color stability found in the present study is that any color alteration occurring in the thin cement layers used in clinical situations is less perceptible than similar discoloration occurring in the thicker layers used in laboratory studies. ${ }^{16,17}$

The color coordinates showed slight variations. Positive $\Delta \mathrm{a}^{*}$ values indicate a reddish color. ${ }^{13,25} \Delta \mathrm{b}^{*}$ presented a change from positive to negative values, which indicates a tendency of yellowing over time. An explanation for the yellowish appearance of a resin material is the presence of Bis-GMA monomer in its formulation. Bis-GMA has an inherent tendency to yellow, especially when exposed to ultraviolet light and heat. ${ }^{1,20}$

Color difference formulas are a valuable instrument to guide the selection of esthetic materials in dentistry. ${ }^{31}$ The CIELab color difference formula has been used for most of the studies evaluating the color of dental materials. ${ }^{23}$ More 
recently, the CIEDE2000 adjusted formula was developed to improve CIELab correction between the computed and perceived color $^{23}$ and better determine the perceptibility and acceptability of dental ceramics. ${ }^{24}$ In this study, both of the color difference formulas were used to allow comparison with previous studies. It is essential to correlate instrumental color difference values with acceptability and perceptibility to provide accurate clinical interpretation, ${ }^{23}$ that is, the clinical relevance of color difference formulas depends on how closely the threshold of color change agrees with human visual judgment. ${ }^{32}$ The clinical acceptability thresholds (the smallest color difference acceptable by $50 \%$ of observers) used in the present study were based on the findings of Ghinea et $\mathrm{al}^{23}$ as they determined thresholds for ceramics, using both the CIELab and the CIEDE2000 formulas.

Across all evaluation periods, a portion of restorations showed $\Delta \mathrm{E}^{*}{ }_{\mathrm{ab}}>3.46$ and $\Delta \mathrm{E}^{*}{ }_{00}>2.25^{23}$ for both the lightand dual-polymerizing modes (Fig. 2). Lu and Powers ${ }^{16}$ also observed that the color alteration of the cement used in this study, with dual- and light-polymerized modes, was considered clinically unacceptable after artificially accelerated aging. Perceptible color alteration of dual-polymerizing disks of the cement was also observed in the studies by Koishi et al, ${ }^{11}$ Tanoue et $\mathrm{al}^{19}$ and Smith et al. ${ }^{15}$ However, because water sorption in the polymer matrix alters the cement refraction index, the color change found in these studies may be attributed in part to the hydrolytic degradation resulting from the specimens' aging during the storage in distilled water. ${ }^{11}$ Another factor may be the thickness of the cement disks used in these studies ( 1 and $2 \mathrm{~mm}$ ). In the clinical environment, only the cement margin is exposed to the oral environment, as the rest of it is covered by the ceramic restoration. Previous studies evaluating the color stability of ceramic disks cemented on substrates found unacceptable color changes for light- and dualpolymerizing cements after thermocycling ${ }^{13}$ and a perceptible color change for dual-polymerizing cements after water storage. ${ }^{12}$

Marginal discoloration was not detected until 1 year after the procedure and then in 10\% of the restorations. The percentage was $35 \%$ at the 2-year color assessment. Water sorption of the resin cement and pigmentation from food and beverages may cause marginal discoloration, ${ }^{8}$ and thus the longer the cement is exposed, the higher the probability of staining. The intake questionnaire showed that the most frequently consumed substances were coffee and a Brazilian beverage, mate, which are possibly related to the marginal discoloration. The presence of marginal staining is in agreement with previous reports. ${ }^{8,30}$ All instances were classified as Bravo, which represents slight staining in a small area. No restoration was classified as Charlie, which represents staining in more than $50 \%$ of the circumferential margin, probably because of the laminate veneers' intimate adaptation to the tooth structure and the fact that the margins were located in a way that provided adequate access for cement finishing and polishing procedures. ${ }^{2}$ Moreover, bonding to enamel is more predictable than to dentin because of its higher mineral content. ${ }^{4}$ Proper adhesion to the tooth structure reduces the possibility of microleakage and severe marginal staining. ${ }^{3.3}$

The present study had some limitations. A standard clinical spectrophotometer was used. Color measurements may be subject to edge loss when such smallaperture devices are used, which is influenced by curved dental surfaces and translucent ceramic measured. ${ }^{34}$ In addition, our findings might not extrapolate to the anterior teeth as they would presumably be more exposed to environmental ultraviolet light, which can induce the oxidation of tertiary amines. ${ }^{16}$ Furthermore, the study group was composed exclusively of dentists, who are possibly more conscientious with their oral hygiene than the general population, which may restrict generalization of the findings. It would be desirable that a wider variety of cements was evaluated using other populations in future studies.

\section{CONCLUSIONS}

Based on the findings of this randomized clinical trial, the following conclusions were drawn:

1. The light- and dual-polymerizing modes presented similar color changes for all evaluated periods $(2,6$, 12 , and 24 months).

2. Color alteration beyond clinically acceptable thresholds was observed for both polymerizing modes. At 24 months, $40 \%$ and $20 \%$ of restorations presented unacceptable color changes for light- and dual-polymerization modes, respectively.

3. Marginal discoloration was observed from 1 year. At 24 months, $40 \%$ and $30 \%$ of veneers presented slight marginal discoloration for light- and dualpolymerization modes, respectively.

\section{REFERENCES}

1. Archegas LR, Freire A, Vieira S, Caldas DB, Souza EM. Colour stability and opacity of resin cements and flowable composites for ceramic veneer luting after accelerated ageing. J Dent 2011;39:804-10.

2. Calamia JR, Calamia CS. Porcelain laminate veneers: reasons for 25 years of success. Dent Clin North Am 2007;51:399-417.

3. Hekimoğlu C, Anil N, Etikan I. Effect of accelerated aging on the color stability of cemented laminate veneers. Int J Prosthodont 2000;13:29-33.

4. Trajtenberg CP, Caram SJ, Kiat-amnuay S. Microleakage of all-ceramic crowns using self-etching resin luting agents. Oper Dent 2008;33:392-9.

5. Okida RC, Filho AJ, Barao VA, Dos Santos DM, Goiato MC. The use of fragments of thin veneers as a restorative therapy for anterior teeth disharmony: a case report with 3 years of follow-up. J Contemp Dent Pract 2012;13: 416-20.

6. Ghavam M, Amani-Tehran M, Saffarpour M. Effect of accelerated aging on the color and opacity of resin cements. Oper Dent 2010;35:605-9.

7. Magalhães APR, Cardoso PC, Souza JB, Fonseca RB, Pires-de Souza FC, Lopez LG. Influence of activation mode of resin cement on the shade of porcelain veneers. J Prosthodont 2014;23:291-5. 
8. Aykor A, Ozel E. Five-year clinical evaluation of 300 teeth restored with porcelain laminate veneers using total-etch and a modified self-etch adhesive system. Oper Dent 2009;34:516-23.

9. Kilinc E, Antonson SA, Hardigan PC, Kesercioglu A. Resin cement color stability and its influence on the final shade of all-ceramics. J Dent 2011;39: e30-6.

10. Chang J, Da Silva JD, Sakai M, Kristiansen J, Ishikawa-Nagai S. The optical effect of composite luting cement on all ceramic crowns. J Dent 2009;37:937-43.

11. Koishi $Y$, Tanoue N, Atsuta M, Matsumura H. Influence of visible-light exposure on colour stability of current dual-curable luting composites. J Oral Rehabil 2002;29:387-93.

12. Nathanson D, Banasr F. Color stability of resin cements-an in vitro study. Pract Proced Aesthet Dent 2002;14:449-55.

13. Almeida JR, Schmitt GU, Kaizer MR, Boscato N, Moraes RR. Resin-based luting agents and color stability of bonded ceramic veneers. J Prosthet Dent 2015;114:272-7.

14. Noie F, O'Keefe KL, Powers JM. Color stability of resin cements after accelerated aging. Int J Prosthodont 1995;8:51-5.

15. Smith DS, Vandewalle KS, Whisler G. Color stability of composite resin cements. Gen Dent 2011;59:390-4.

16. Lu H, Powers JM. Color stability of resin cements after accelerated aging. Am J Dent 2004:17:354-8

17. Berrong JM, Weed RM, Schwartz IS. Color stability of selected dual-cure composite resin cements. J Prosthodont 1993;2:24-7.

18. Turgut $\mathrm{S}$, Bagis B. Colour stability of laminate veneers: an in vitro study. J Dent 2011;39:e57-64.

19. Tanoue N, Koishi Y, Atsuta M, Matsumura H. Properties of dual-curable luting composites polymerized with single and dual curing modes. J Oral Rehabil 2003:30:1015-21.

20. Ferracane JL, Moser JB, Greener EH. Ultraviolet light-induced yellowing of dental restorative resins. J Prosthet Dent 1985;54:483-7.

21. Hedrick VE, Comber DL, Estabrooks PA, Savla I, Davy BM. The beverage intake questionnaire: determining initial validity and reliability. J Am Diet Assoc 2010;110:1227-32.

22. Viera AJ, Garrett JM. Understanding interobserver agreement: the kappa statistic. Fam Med 2005;37:360-3.

23. Ghinea R, Pérez MM, Herrera LJ, Rivas MJ, Yebra A, Paravina RD. Color difference thresholds in dental ceramics. J Dent 2010;38:e57-64.

24. Gómez-Polo C, Muñoz MP, Lorenzo Luengo MC, Vicente P, Galindo P, Martín Casado AM. Comparison of the CIELab and CIEDE2000 color difference formulas. J Prosthet Dent 2016;115:65-70.

25. Albuquerque PP, Moreira AD, Moraes RR, Cavalcante LM, Schneider LF Color stability, conversion, water sorption and solubility of dental composites formulated with different photoinitiator systems. J Dent 2013;41: e67-72.

26. Sharma G, Wu W, Dalal EN. The CIEDE2000 color-difference formula: implementation notes, supplementary test data, and mathematical observations. Color Res Appl 2005;30:21-30.

27. Cvar JF, Ryge G. Reprint of criteria for the clinical evaluation of dental restorative materials. 1971. Clin Oral Investig 2005;9:215-32.

28. Moncada G, Fernández E, Martín J, Arancibia C, Mjör IA, Gordan VV. Increasing the longevity of restorations by minimal intervention: a two-year clinical trial. Oper Dent 2008;33:258-64.

29. Sideridou I, Tserki V, Papanastasiou G. Study of water sorption, solubility and modulus of elasticity of light-cured dimethacrylate-based dental resins. Biomaterials 2003;24:655-65.

30. Guess PC, Selz CF, Voulgarakis A, Stampf S, Stappert CF. Prospective clinical study of press-ceramic overlap and full veneer restorations: 7-year results. Int J Prosthodont 2014;27:355-8.

31. Paravina RD, Ghinea R, Herrera LJ, Bona AD, Igiel C, Linninger M, Sakai M, Takahashi H, Tashkandi E, Perez Mdel M. Color difference thresholds in dentistry. J Esthet Restor Dent 2015;27:S1-9.

32. Khashayar G, Bain PA, Salari S, Dozic A, Kleverlaan CJ, Feilzer AJ. Perceptibility and acceptability thresholds for colour differences in dentistry. J Dent 2014;42:637-44.

33. Borges MA, Matos IC, Dias KR. Influence of two self-etching primer systems on enamel adhesion. Braz Dent J 2007;18:113-8.

34. Pop-Ciutrila IS, Ghinea R, Colosi HA, Dudea D. Dentin translucency and color evaluation in human incisors, canines, and molars. J Prosthet Dent 2016;115:475-81.

\section{Corresponding author:}

Dr Ana Maria Estivalete Marchionatti

Department of Restorative Dentistry

Federal University of Santa Maria

Floriano Peixoto, 1184

97015-373, Santa Maria, RS

BRAZIL

Email: anamarchionatti@hotmail.com

\section{Acknowledgments}

The authors thank Gilberto Venturini for fabricating the laminate veneers and Vita Zahnfabrik for lending the Vita Easyshade device.

Copyright (C) 2016 by the Editorial Council for The Journal of Prosthetic Dentistry. 